\title{
Revisão Sistemática da Literatura sobre o uso de Learning Analytics no ensino de programação
}

\author{
José Wilker Pereira Luz, IFMA, josewilkerluz@ifma.edu.br \\ https://orcid.org/0000-0002-2057-5229 \\ Márcia Jussara Hepp Rehfeldt, UNIVATES, mrehfeld@univates.br \\ https://orcid.org/0000-0002-0007-8639 \\ Maria Claudete Schorr, UNIVATES, mclaudetesw@univates.br \\ https://orcid.org/0000-0002-1874-7917
}

\begin{abstract}
Resumo: As técnicas de Learning Analytics (LA) são usadas numa variedade de níveis educacionais, com objetivos diferentes, o que permite descrever, diagnosticar, prever e prescrever a aprendizagem, especialmente, no Ensino Superior. Para entender o uso do LA, analisamos, neste artigo, através de uma revisão sistemática da literatura, os procedimentos de pesquisa e os resultados produzidos, que tratam da aplicação da LA no ensino de programação. Nesse sentido, definimos questões de pesquisa para compreender o seu impacto nos processos de ensino e aprendizagem. Essas nos permitiram avaliar que as teorias de aprendizagem não foram utilizadas explicitamente, os processos metodológicos mais usados e como os dados são coletados e analisados.
\end{abstract}

Palavras-Chave: Learning Analytics. Ensino de Programação. Revisão Sistemática da Literatura.

\section{Systematic Literature Review on the Use of Learning Analytics in Programming Education}

Abstract: Learning Analytics (LA) techniques are used at a variety of educational levels and different goals, which allow you to describe, diagnose, predict and prescribe learning, especially in Higher Education. To understand the use of LA, in this article, we analyze, through a systematic review of the literature, the research procedures and the results produced that deal with the application of LA in teaching programming. Thus, we define research questions to understand their impact on teaching and learning processes. These allowed us to assess that learning theories were not used explicitly, the most used methodological processes, and how data is collected and analyzed.

Keywords: Learning Analytics, Programming Education, Systematic Literature Review

\section{Introdução}

As técnicas de Learning Analytics (LA) têm se destacado como alternativa para compreender o comportamento e a performance dos alunos durante o processo educacional. Esta técnica analisa dados educacionais obtidos a partir da interação do aluno com recursos online e da utilização dos dados disponíveis nos Sistemas de Gestão de Aprendizagem (Learning Management System - LMS) (FREITAS, 2019). Para entender o uso do LA, analisamos, neste trabalho, os procedimentos de pesquisa e os resultados produzidos por teses e dissertações e artigos publicados em revistas ou conferências que tratam da aplicação da LA no ensino de programação, bem como seu impacto nos processos de ensino e de aprendizagem. Este artigo está organizado do 
seguinte modo: A seção 2 descreve a metodologia adotada neste estudo. As seções 3 e 4 relatam a síntese, a análise e as discussões dos resultados. A seção 4 apresenta as considerações deste estudo.

\section{Metodologia}

Para a realização desta pesquisa, utilizamos o método de Revisão Sistemática de Literatura (RSL), conforme guia descrito em (KITCHENHAM, B. et al, 2007). Os autores apresentam diretrizes para a realização de revisões sistemáticas rigorosas na busca por evidências empíricas atuais na comunidade acadêmica. Este guia é utilizado principalmente em pesquisas na área da ciência da computação. O objetivo desta revisão é analisar os procedimentos de pesquisa e os resultados produzidos por teses e dissertações e artigos publicados em revistas ou conferências que tratam da aplicação da LA no ensino de programação e o seu impacto nos processos de ensino e de aprendizagem. A seção a seguir apresenta o protocolo de pesquisa.

\subsection{Protocolo de Pesquisa}

\subsubsection{Questões de Pesquisa}

Para orientar o estudo, foi definida a seguinte questão de pesquisa: Como a $L A$ tem sido aplicada no ensino de programação? Para apoiar a análise de dados, as seguintes subquestões foram definidas: a) Quais as principais teorias de aprendizagem que embasam a aplicação nos processos de ensino e de aprendizagem? Como se estruturam os processos metodológicos? Como os dados do ensino de programação foram coletados e analisados?

\subsubsection{Estratégia de Busca dos Estudos Primários}

Para alcançar um conjunto relevante de estudos, as seguintes decisões foram necessárias para guiar a RSL:

a Fontes de Informação - Utilizamos as bases de busca Elsevier Scopus ${ }^{1}, A C M$ Digital Library ${ }^{2}$, ScienceDirect ${ }^{3}$ e IEEE Digital Library ${ }^{4}$, acessadas através do site de Periódicos da CAPES ${ }^{5}$, que nos permitiram identificar estudos apresentados em conferências ou publicados em periódicos vinculados aos principais repositórios da área de Ciência da Computação. Utilizamos também como base de busca, a Biblioteca Digital Brasileira de Teses e Dissertações - BDTD ${ }^{6}$.

b Palavras-Chave - As palavras-chave que delimitaram o escopo da RSL foram: LA, Ensino de Programação, Aprendizagem em Programação. Assim, para contemplar os trabalhos inseridos nas interseções desses campos de pesquisa, foram elaboradas as seguintes Strings de busca em inglês (("learning programming" OR "teaching programming" OR "programming education") AND

\footnotetext{
${ }^{1} \mathrm{https}: / /$ www.scopus.com/

${ }^{2}$ https://dl.acm.org/

${ }^{3} \mathrm{https}: / /$ www.sciencedirect.com/

${ }^{4} \mathrm{https} / / /$ ieeexplore.ieee.org/Xplore/home.jsp

${ }^{5} \mathrm{https}: / / \mathrm{www}-$ periodicos-capes-gov-br.ezl.periodicos.capes.gov.br/index.php?

${ }^{6} \mathrm{https}: / / \mathrm{bdtd}$.ibict.br/vufind/
} 
("Learning Analytics")) e português (("aprendizagem em programação" OR "Ensino de programação") AND ("Learning Analytics")).

c Critérios de Inclusão e de Exclusão - Para a inclusão na nossa RSL, o trabalho deveria inserir pesquisas primárias sobre o uso da LA nos Processos de Ensino e de Aprendizagem de Programação de Computadores. Como critérios de exclusão, utilizamos os seguintes: artigos que não estivessem na língua inglesa ou portuguesa; pesquisas duplicadas; pesquisas secundárias; pesquisas não disponíveis de forma gratuita; artigos curtos (Short Papers); artigos que não apresentassem intervenção no ensino de programação. Caso se enquadrasse em pelo menos um dos critérios de exclusão, o trabalho foi classificado como excluído.

\subsection{Avaliação da Qualidade}

As pesquisas incluídas nesta revisão foram verificadas em relação à sua qualidade, cuja avaliação foi orientada com base nos seguintes critérios: a pesquisa apresenta uma definição clara dos objetivos? (Q1); a pesquisa apresenta uma contextualização dos usuários? (Q2); a pesquisa apresenta uma descrição do contexto experimental? (neste estudo está relacionado ao ensino de programação) (Q3); o estudo deixa claras as limitações? (Q4). Para cada estudo, a adequação a cada critério foi classificada em sim $(\mathrm{S})$, não $(\mathrm{N})$ e parcialmente $(\mathrm{P})$. Por exemplo, estudos que descreveram apenas o nível de ensino e as disciplinas cursadas pelos participantes, mas não mencionaram o conhecimento prévio em programação e outras informações relevantes receberam a classificação "parcialmente" para Q2.

\subsection{Execução da Pesquisa}

A execução da pesquisa ocorreu a partir dos trabalhos identificados por meio das Strings de busca e das palavras-chave, limitados ao período entre 2011 e 2021 e aos tipos de documentos, "Conference Paper", "Article", "Tese" e "Dissertação". Após a execução das buscas nas bases de dados, exportamos os metadados das pesquisas em formato bib para a ferramenta de gestão de revisão sistemática da literatura Parsifal ${ }^{7}$. O processo de seleção dos trabalhos ocorreu em duas etapas. Na primeira, foram analisados o título, o resumo e as palavras-chave, para encontrar pesquisas que atendessem aos critérios de inclusão. Na segunda etapa, foi realizada uma leitura completa dos trabalhos selecionados, aplicando os critérios de exclusão, além de verificar novamente os critérios de inclusão, o que permitiu a seleção final das pesquisas que respeitaram os critérios estabelecidos.

\section{Análise dos Resultados}

As Strings de busca disponíveis no item (2.1.1), item b, permitiram encontrar 192 (cento e noventa e duas) pesquisas (Figura 1), 24 (vinte e quatro) artigos na base Elsevier Scorpus, 41 (quarenta e um) artigos na base ACM Library, 49 (quarenta e nove) artigos

\footnotetext{
${ }^{7}$ A Parsifal é uma ferramenta online desenvolvida para apoiar pesquisadores na realização de revisões sistemáticas da literatura, fornece uma maneira de documentar todo o processo e ajuda a lembrar o que foi importante durante uma RSL. Disponível em: https://parsif.al/ acesso em: 31 de março de 2021.
} 
na base ScienceDirect e 18 (dezoito) artigos na base IEEE Digital Library. Em relação às teses e dissertações da base Biblioteca Digital de Teses e Dissertações - BDTD, a String de busca, conforme formatada no item (2.1.1), item b, não gerou nenhum resultado. Decidimos, então, fazer a busca pelas palavras-chave, na base BDTD, o que gerou uma lista de 11 (onze) teses e 26 (vinte seis) dissertações.

Figura 1: Resumo do Processo da RSL

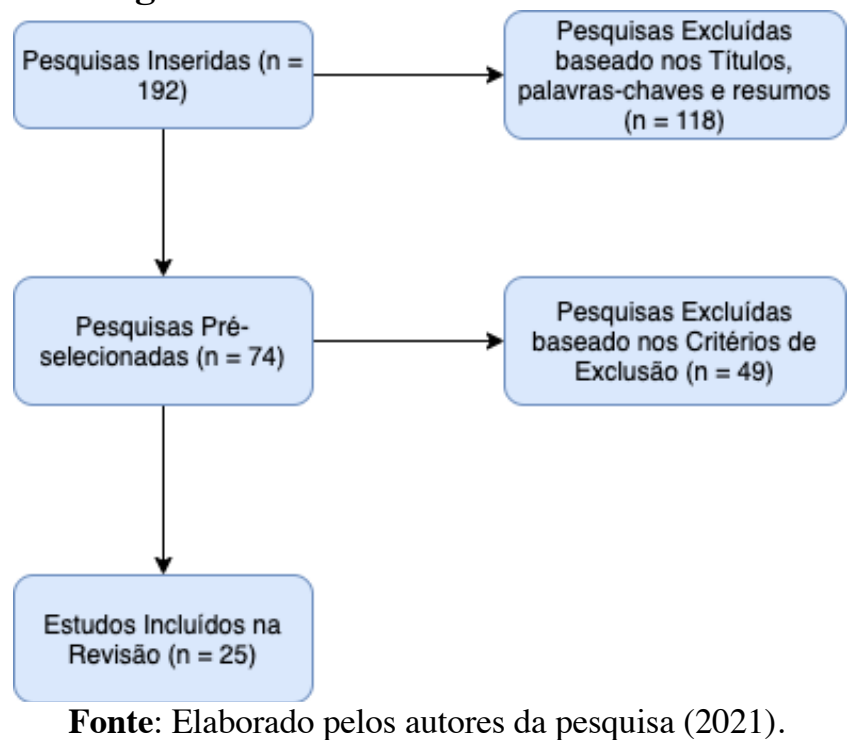

Após a primeira etapa de seleção, baseada na leitura do título, das palavras-chave e dos resumos, aplicamos o critério de inclusão, que permitiu filtrar a busca para selecionar os que mais se aproximavam da nossa proposta de investigação. Nesta fase, foram descartadas da base BDTD, sete (7) teses e 18 (dezoito) dissertações. Das bases Scorpus, ACM, Science Direct e IEEE, foram descartadas 59 (cinquenta e nove) e permaneceram 74 (setenta e quatro) pesquisas pré-selecionadas (Figura 1).

Em seguida, aplicamos os critérios de exclusão, que permitiram filtrar mais ainda a pesquisa. Nesta etapa, foram encontradas dez (10) pesquisas duplicadas, duas (2) pesquisas secundárias, cinco (5) pesquisas não disponíveis de forma gratuita e 18 (dezoito) artigos curtos (Short paper). Com exceção das teses e dissertações, todas as outras pesquisas estavam escritas em língua inglesa. Após a aplicação dos critérios de inclusão e de exclusão, 25 (vinte e cinco) estudos foram incluídos para a leitura completa, sendo quatro (4) teses, oito (8) dissertações e 13 (treze) artigos completos.

No geral, o LA foi usado numa variedade de níveis educacionais e objetivos diferentes, por exemplo, para monitoramento e análise (AFONSO, 2017), (SEDRAZ, 2018), (JUNIOR, 2018), (SILVA, 2017), (ORLANDO, 2018), (DE; BRITO, 2019), (DIANA et al., 2017), (FONSECA; MACEDO; MENDES, 2016), (KRUG; SIMÃO; BASTOS, 2019), (FU et al., 2017); para previsão e intervenção (SEDRAZ, 2018), (FONTES, 2017), (DOMINGOS, 2016), (BRASIL, 2019), (AMO et al., 2018); para tutoria, mentoria, avaliação e feedback (SEDRAZ, 2018), (JUNIOR, 2018), (CARNEIRO, 2018), (HSIAO; HUANG; MURPHY, 2020), (PAREDES; HSIAO; LIN, 2018), (GOMEZ; MORESI; BENOTTI, 2019); para adaptação, personalização e recomendação (SEDRAZ, 2018), (AZCONA; HSIAO; SMEATON, 2019), (PAREDES; HSIAO; LIN, 2018), (KESSELBACHER; BOLLIN, 2019); para reflexão sobre o comportamento dos alunos nos processos de ensino (ROCHA NETO, 2019), (FILVÀ et 
al., 2019). A seguir, apresentamos a aplicação dos critérios de qualidade disponíveis no item 2.2 .

Todas as pesquisas fazem uma apresentação clara dos objetivos (Q1). Quanto à contextualização dos sujeitos da pesquisa (Q2), 70\% das pesquisas não fazem um detalhamento completo, apenas descrevem o quantitativo, o nível de ensino ou as disciplinas cursadas pelos participantes. Em relação ao contexto experimental (Q3), 54\% estão relacionadas ao ensino presencial, $33 \%$ são aplicadas na modalidade a distância e o restante não define a modalidade de aplicação. Ainda, em relação ao contexto experimental (Q3), 84\% das pesquisas usam as disciplinas na área de programação para a coleta de dados, análise e intervenção; os outros 16\% apenas usam dados gerais coletados nos ambientes virtuais de aprendizagem. Por fim, 54\% usam o LMS Moodle para o gerenciamento da aprendizagem e a coleta dos dados. Ademais, $62 \%$ apresentaram limitações da pesquisa (Q4). A seguir, os resultados serão apresentados de acordo com a ordem das questões de pesquisa descritas no item 2.1.1.

3.1.1. Quais as principais teorias de aprendizagem que embasam a aplicação nos processos de ensino e de aprendizagem?

Quanto à utilização de uma teoria de aprendizagem para nortear as discussões e inferências das pesquisas analisadas, constatou-se que nenhum trabalho utilizou teorias de aprendizagem. Porém, conforme argumentam (HUNDHAUSEN; OLIVARES; CARTER, 2017, p. 15),

explicada ou não, alguma teoria de aprendizagem subjacente deve ser aplicada ao projeto de qualquer intervenção. As teorias de aprendizagem tendem a ser bastante amplas: elas descrevem como as pessoas aprendem e quais as condições que melhor promovem a aprendizagem.

\subsubsection{Quais referenciais teóricos embasam a pesquisa?}

Dentre outros aportes teóricos, todas as teses e dissertações analisadas fazem uso dos trabalhos de (SIEMENS; BAKER, 2012) e (CHATTI et al., 2012), para a fundamentação teórica do Learning Analytics. Os primeiros autores apresentam as discussões sobre a origem do LA, sua definição e principais ferramentas e métodos utilizados, ao fazerem a comparação do termo com a mineração de dados educacionais. O segundo grupo de autores apresenta o modelo de referência que nos ajuda a entender melhor o que está envolvido na LA e seus objetivos de uso. A LA é usada em diferentes contextos para diferentes finalidades: Afonso (2017) analisa como os dados históricos de cursos já realizados na modalidade a distância em um AVA podem identificar possíveis problemas no Design Instrucional; Fontes (2012) desenvolve um sistema para monitoramento e avaliação das atividades de tutoria a distância em AVAs, usando o aprendizado de máquina; Sedraz (2018) usa o LA para analisar a Autorregulação da Aprendizagem de alunos em Sala de Aula Invertida; Junior (2018) desenvolveu um framework, para análise de dados multimodais, Multimodal Learning Analytics, fundamentado na abordagem ECD (Evidency Center Design); As pesquisas de (HSIAO; HUANG; MURPHY, 2020), (PAREDES; HSIAO; LIN, 2018) e (PAREDES; HSIAO; LIN, 2018) se fundamentam em (HATTIE e TIMPERLEY, 2007), para desenvolver propostas de análise da aprendizagem, nas quais os alunos recebem feedback formativo e somático, após a realização de atividades. 


\subsubsection{Como se estruturam os processos metodológicos?}

Quanto aos percursos metodológicos, observamos uma diversidade de métodos utilizados pelos investigadores nos estudos analisados, a fim de alcançarem os objetivos almejados. A abordagem quantitativa é usada em $64 \%$ das pesquisas; já a abordagem qualitativa corresponde a $20 \%$ do total dos trabalhos analisados. No entanto, $16 \%$ das pesquisas caracaterizaram-se como quantiqualitativas, o que consideramos ser o ideal para pesquisas que buscam analisar, interpretar e intervir nos processos de aprendizagem através de LA, conforme afirmam (SANDRO GOMES; ARAÚJO GOMES, 2020, p. 78):

Quando realizamos estudos quantiquali, é possível perceber relações entre algumas variáveis. A partir dessa identificação inicial, subsequentes estudos quantitativos podem suplantar a verificação das relações uma a uma, complementando o que antecipadamente fora identificado de forma qualitativa. É o caso, por exemplo, de uma pesquisa que inicia com um levantamento exploratório. A validação e a generalização da etapa qualitativa são circunstanciais, mas, em alguns tipos de pesquisa, uma excelente interpretação narrativa qualitativa que aprofunde o entendimento dos dados quantitativos é muitas vezes mais informativa que o entendimento de relações entre variáveis isoladas (PINK et al., 2017). Os estudos qualitativos permitem inferir estruturas subjacentes de relações entre variáveis, a partir de uma grande quantidade de indícios. No caso particular dos estudos de processos cognitivos, eles permitem realizar inferências sobre os significados que constroem os sujeitos (DA SILVA, 1998). Dessa forma, o processo qualitativo ajuda a esclarecer pontos obscuros revelados pelos dados.

Sobre os métodos de pesquisa, destacamos o trabalho de (SEDRAZ, 2018, p.73), cujo estudo é orientado pelo Design Science Research..$^{8} \mathrm{O}$ autor da pesquisa descreve as diretrizes do método em seis etapas:

a primeira etapa consiste na identificação dos aspectos que motivam a realização do estudo; a segunda diz respeito à definição dos objetivos, os quais podem ser relacionados com resultados quantitativos ou qualitativos; as três etapas seguintes são destinadas à geração e aos testes do artefato que auxiliará na solução do problema; os resultados de um ciclo dessas três etapas podem ser utilizados para, iterativamente, subsidiar a geração de novos artefatos. Por fim, na etapa de comunicação, deverá ser evidenciado o rigor com o qual a pesquisa foi conduzida, assim como o quão eficaz foi a solução encontrada.

\subsubsection{Como os dados do ensino de programação foram coletados e analisados?}

De acordo com Chatti et al., (2012), no processo de LA, do ponto de vista técnico, o primeiro passo é coletar dados provenientes de diversos ambientes e sistemas. O passo seguinte é a análise e a ação, que envolvem empregar os métodos de LA para analisar os dados de acordo com os objetivos firmados. Nesse sentido, descrevemos abaixo como foram realizadas as coletas de dados e as análises nas pesquisas selecionadas.

Afonso (2017) coletou os dados históricos das disciplinas de lógica de programação e de programação para dispositivos móveis, disponíveis no LMS Moodle, em edições anteriores, além dos dados gerados durante a execução das mesmas disciplinas (observação, critérios ergonômicos, questionário de avaliação, gravação de vídeo e

\footnotetext{
${ }^{8}$ PEFFERS et al., 2007
} 
análise de carga mental). Os dados foram tratados para a definição de variáveis e analisados em duas fases: primeiro, por meio da utilização de estatística descritiva e depois, por meio do teste de hipóteses, com o objetivo de identificar de forma rápida possíveis problemas no Design Instrucional (DI) de um curso, a partir de seus dados históricos.

Junior (2018) incluiu nos jogos digitais um conjunto de agentes inteligentes de software, na forma de uma biblioteca, cujas funções são acionadas de dentro do jogo, para capturar os dados do jogador jogando, inclusive seus dados psicofisiológicos, através de sensores (filmagens dos jogadores jogando). Para o tratamento e a integração dos dados multimodais, a pesquisa utilizou técnicas de Inteligência artificial. Ademais, fez a análise, com base em métodos e técnicas de LA, para consultas e emissão de relatórios, a partir dos dados coletados e tratados.

Diana et al. (2017), usando os dados da avaliação de códigos de programação desenvolvidos pelos alunos, converteram os dados de log brutos, numa série de estados de código. Em seguida, treinaram modelos preditivos para prever as notas dos alunos e, por fim, integraram esses modelos preditivos a um painel do instrutor (dashboard) e estimaram a utilidade do painel em sala de aula.

Gomez et al. (2019) apresentaram uma ferramenta de codificação online, que registra o processo de aprendizagem do aluno durante as atividades de programação, lançando cada envio feito pelo aluno, junto com o feedback gerado automaticamente. A pesquisa analisa como as crianças criam programas numa linguagem de programação baseada em texto, bem como avalia os alunos em conceitos fundamentais de programação, envolvendo interpretação de código.

Nas pesquisas de (FONSECA; MACEDO; MENDES, 2016), (KRUG; SIMÃO; BASTOS, 2019), (FU et al., 2017), (KESSELBACHER; BOLLIN, 2019), os dados são coletados dos códigos de programação desenvolvidos pelos alunos. Para isso, foram utilizados plug-ins integrados aos ambientes de desenvolvimento (Integrated Development Environment - IDE) e ao LMS Moodle. Essas ferramentas, além da coleta, geram a análise dos dados. Para isso, fazem a compilação dos códigos dos alunos para verificação de possíveis erros e acertos em relação à sintaxe e à semântica da programação. Na sequência, apresentam a análise através de dashboard aos professores, que poderão utilizar as informações para a identificação de desvios do caminho normal a ser seguido e o fornecimento de um feedback ao aluno.

A plataforma Web-based Programming Grading Assistant (WPGA) é utilizada para a coleta de dados nas pesquisas de (HSIAO; HUANG; MURPHY, 2020), (PAREDES; HSIAO; LIN, 2018), (AZCONA; HSIAO; SMEATON, 2019), (PAREDES; HSIAO; LIN, 2018). A tecnologia coleta os dados por meio da digitalização das avaliações, baseada em papel. Em seguida, faz a análise preditiva multimodal, que fornece a previsão em tempo real. Por fim, entrega um feedback dinâmico aos alunos, com base em previsões de desempenho e sugestões de código dos melhores alunos da classe.

A ferramenta Clickstream é usada por (AMO et al., 2018), que avaliam o comportamento dos alunos num curso de Scratch, por meio da captura de cliques e do pressionamento das teclas do teclado, usando a análise preditiva, correlacionada às avaliações realizadas pelos professores. FILVÀ et al (2019) também usam a ferramenta Clickstream nas práticas de programação e mostram como a taxonomia de comportamentos de programação pode ser facilmente usada numa sala de aula, com o objetivo de ajudar os professores a compreender como os alunos se comportam em sua 
prática e, consequentemente, melhorar a avaliação e o acompanhamento dos alunos para evitar reprovações.

\section{Discussões}

Analisando os aportes teóricos das investigações examinadas, verificamos que nenhum estudo recorreu aos pressupostos de Teorias da Aprendizagem, associados às pesquisas em LA. Esse fato chamou nossa atenção e despertou o nosso interesse por teorias que possam acompanhar a aprendizagem individual e coletiva dos alunos. Nesse contexto, conforme o trabalho de Hundhausen et al (2017), defendemos que as teorias de aprendizagem devem ser aplicadas junto com as técnicas de LA, pois são de suma importância para as investigações sobre o comportamento individual e coletivo dos alunos, com a finalidade de compreender os indícios de aprendizagem gerados a partir da análise dos dados numa disciplina de programação.

Constatamos que as metodologias empregadas em cada estudo foram fundamentais no seu delineamento e desenvolvimento, mostrando o quanto é difícil desenvolver uma pesquisa, principalmente em nível de doutoramento, pois o pesquisador deverá estar preparado e atento para superar diversos desafios que encontrará ao longo dos estudos. Para a obtenção de dados fidedignos ao ir a campo buscar respostas acerca de uma problemática, é de fundamental importância seguir um percurso metodológico seguro, mesmo que este esteja em constante construção ao longo do estudo.

A partir das pesquisas acima, pôde-se observar que os métodos e ferramentas serviram para coletar e analisar dados de eventos gerados durante o desenvolvimento de códigos de programação, o que permitiu o mapeamento de padrões de comportamento de cada indivíduo de um grupo observado, com a finalidade de auxiliar na análise da sua evolução em atividades de programação, procurando assim trazer possibilidades de aplicação nos processos de ensino e de aprendizagem, além de auxiliar o professor na elaboração de estratégias, materiais e métodos para o ensino de programação.

\section{Considerações}

Ao analisar as teses, dissertações e artigos pesquisados, percebemos que as pesquisas têm se concentrado na identificação de padrões de comportamento, com base nos dados gerados nos processos de ensino e de aprendizagem em programação, fundamentados na geração de modelos e no desenvolvimento de ferramentas para organizar o uso da LA. No geral, os trabalhos buscam melhorar o feedback e o fornecimento de mecanismos de incentivo, através do aprimoramento das mensagens de erro de sintaxe e semântica, tornando-as mais compreensíveis, além da geração de dicas com base nos padrões extraídos dos dados de programação. Além disso, a maioria das pesquisas é orientada por uma abordagem quantitativa, com a ilustração dos fenômenos através de estudos experimentais e correlacionais. No entanto, não são orientadas por teorias de aprendizagem, o que julgamos ser fundamental durante os processos de intervenção no ensino. Logo, podemos inferir que é uma preocupação sujeita a novas investigações que visem compreender e traçar metodologias de ensino que contribuam para a melhoria do ensino e/ou da aprendizagem, usando LA.

\section{Referências}


AFONSO, D. Visualização de Dados como Suporte ao Design Instrucional. 2017. AMO, D. et al. Learning analytics to assess students' behavior with scratch through clickstream. CEUR Workshop Proceedings, v. 2188, p. 74-82, 2018. AZCONA, D.; HSIAO, I. H.; SMEATON, A. F. Personalizing computer science education by leveraging multimodal learning analytics. Proceedings - Frontiers in Education Conference, FIE, v. 2018- Octob, p. 1-9, 2019. BRASIL, P. C. Um processo analítico de dados educacionais: uma abordagem baseada nos dados socioeconômicos e educacionais dos alunos. [s.1.] Brasil, 1 nov. 2019. Disponível em: <https://repositorio.ufrn.br/jspui/handle/123456789/28336>. Acesso em: 10 set. 2020.

CARNEIRO, J. DO N. Aplicação de Learning Analytics para Modelagem do Aluno de acordo com a Taxonomia de Bloom Revisada. [s.1.] Universidade Federal da Paraíba, 21 jun. 2018. Disponível em:

<https://repositorio.ufpb.br/jspui/handle/123456789/14483>. Acesso em: 10 set. 2020.

DE, M. T.; BRITO, S. UM PLUGIN DO TIPO REPORT PARA A IDENTIFICAÇÃO DO RISCO DE EVASÃO NA EDUCAÇÃO SUPERIOR A DISTÂNCIA QUE USA TÉCNICAS DE VISUALIZAÇÃO DE DADOS. [s.l.] Universidade Federal da Paraíba, 17 jan. 2019. Disponível em: <https://repositorio.ufpb.br/jspui/handle/123456789/15990>. Acesso em: 10 set. 2020. DIANA, N. et al. An instructor dashboard for real-time analytics in interactive programming assignments. ACM International Conference Proceeding Series, p. 272-279, 2017.

DOMINGOS, E. D. O. S. MODELO DE DIAGNÓSTICO DE DIFICULDADES DE APRENDIZAGEM ORIENTADO A CONCEITOS. [s.1.] Universidade Federal da Paraíba, 29 fev. 2016. Disponível em: $<$ https://repositorio.ufpb.br/jspui/handle/tede/9253>. Acesso em: 10 set. 2020. FILVÀ, D. A. et al. Clickstream for learning analytics to assess students' behavior with Scratch. Future Generation Computer Systems, v. 93, p. 673-686, 2019.

FONSECA, N. G.; MACEDO, L.; MENDES, A. J. CodeInsights: Monitoring Programming Students' Progress. Proceedings of the 17th International Conference on Computer Systems and Technologies 2016. Anais...: CompSysTech '16.New York, NY, USA: Association for Computing Machinery, 2016Disponível em: <https://doi.org/10.1145/2983468.2983492> FONTES, L. MONITUM: Um Sistema Proativo para Monitoramento e Avaliação das Atividades de Tutoria a Distância em AVAs. [s.1.] Brasil, 10 mar. 2017. Disponível em: <https://repositorio.ufrn.br/jspui/handle/123456789/23310>. Acesso em: 10 set. 2020.

FU, X. et al. Real-Time Learning Analytics for C Programming Language Courses. Proceedings of the Seventh International Learning Analytics \& Knowledge Conference. Anais...: LAK '17.New York, NY, USA: Association for Computing Machinery, 2017Disponível em: <https://doi.org/10.1145/3027385.3027407> GOMEZ, M. J.; MORESI, M.; BENOTTI, L. Text-Based Programming in Elementary School: A Comparative Study of Programming Abilities in Children with and without Block-Based Experience. Proceedings of the 2019 ACM Conference on Innovation and Technology in Computer Science Education. Anais...: ITiCSE '19.New York, NY, USA: Association for Computing Machinery, 2019Disponível em: <https://doi.org.ez364.periodicos.capes.gov.br/10.1145/3304221.3319734> 
HSIAO, I. H.; HUANG, P. K.; MURPHY, H. Integrating Programming Learning Analytics Across Physical and Digital Space. IEEE Transactions on Emerging Topics in Computing, v. 8, n. 1, p. 206-217, 2020.

HUNDHAUSEN, C. D.; OLIVARES, D. M.; CARTER, A. S. IDE-Based Learning Analytics for Computing Education: A Process Model, Critical Review, and Research Agenda. ACM Trans. Comput. Educ., v. 17, n. 3, ago. 2017. JUNIOR, H. A. P. AVALIAÇÃO DA APRENDIZAGEM EM JOGOS DIGITAIS BASEADA EM LEARNING ANALYTICS SOBRE DADOS MULTIMODAIS VITÓRIA 2018. [s.1.] Universidade Federal do Espírito Santo, 20 dez. 2018. Disponível em: <http://repositorio.ufes.br/handle/10/10730>. Acesso em: 10 set. 2020. KESSELBACHER, M.; BOLLIN, A. Discriminating Programming Strategies in Scratch: Making the Difference between Novice and Experienced Programmers. Proceedings of the 14th Workshop in Primary and Secondary Computing Education. Anais...: WiPSCE'19.New York, NY, USA: Association for Computing Machinery, 2019Disponível em:

<https://doi.org.ez364.periodicos.capes.gov.br/10.1145/3361721.3361727>

KRUG, D. L.; SIMÃO, J. M.; BASTOS, L. C. Method and tools for mapping the evolution of programmers during the development of computer programs. ACM

International Conference Proceeding Series, p. 491-500, 2019.

PAREDES, Y. V.; HSIAO, I. H.; LIN, Y. Personalized guidance on how to review paper-based assessments. ICCE 2018 - 26th International Conference on Computers in Education, Main Conference Proceedings, p. 257-265, 2018.

SANDRO GOMES, A.; ARAÚJO GOMES, C. R. Classificação dos tipos de pesquisa em Informática na Educação. Metodologia de Pesquisa Científica em Informática na Educação: Concepção de Pesquisa, 2020.

SEDRAZ, J. C. S. Uma Abordagem de Learning Analytics para a Autorregulação da Aprendizagem de Estudantes em Sala de Aula Invertida. 2018.

SILVA, V. VLA DASHBOARD: UM MECANISMO PARA VISUALIZAÇÃO DO DESEMPENHO DOS ESTUDANTES DE MATEMÁTICA NO ENSINO MÉDIO. [s.1.] Universidade Federal do Amazonas, 24 ago. 2017. Disponível em: $<$ https://tede.ufam.edu.br//handle/tede/5959>. Acesso em: 10 set. 2020. 